[Vicino Oriente XXV (2021), pp. 143-165]

\title{
LO STUDIO DEL CORPUS CERAMICO DELLA CITTADELLA DI ERBIL E DEI CONTESTI ARCHEOLOGICI DI RITROVAMENTO: RISULTATI PRELIMINARI
}

\author{
Luca Colliva - MAIKI \& Alma Mater Studiorum - University of Bologna \\ Serenella Mancini - MAIKI \& Sapienza University of Rome ${ }^{1}$
}

The paper presents the preliminary results of the joint HCECR-MAIKI study of the ceramic corpus found during the HCECR excavations at the Erbil Citadel and explores its relationships with the archaeological stratigraphy.

Keywords: archaeology; Erbil Citadel; Iraqi Kurdistan; Islamic Pottery; stratigraphy

\section{MAIKI - MiSSIONE ARCHEOLOGICA ITALIANA NEL KURDISTAN IRACHENO ${ }^{2}$}

La Missione Archeologica Italiana nel Kurdistan Iracheno (MAIKI) del Dipartimento di Scienze dell'Antichità della Sapienza - Università di Roma, diretta dal Prof. Carlo Giovanni Cereti, nasce nel 2011 con l'obiettivo di studiare lo sviluppo storico, politico e culturale della regione curda in epoca storica.

La MAIKI è la naturale continuazione delle attività di salvaguardia e ricerca storicofilologica e archeologiche realizzate da un gruppo di ricerca italiano, anch'esso diretto dal Prof. C.G. Cereti, attivo nella Regione Autonoma del Kurdistan Iracheno (KRG) dal 2006 nell'ambito dei progetti realizzati nell'area dall'Istituto Italiano per l'Africa e l'Oriente (IsIAO) e della Sapienza ${ }^{3}$.

La MAIKI, operando in stretta collaborazione con le istituzioni locali preposte alla conservazione, gestione e valorizzazione del patrimonio culturale della regione autonoma del Kurdistan Iracheno, come l'Alta Commissione per la Rivitalizzazione della Cittadella di Erbil (High Commission for the Erbil Citadel Revitalization - HCECR), la Direzione Generale delle Antichità del Governo Regionale Curdo (KRG) e le Direzioni delle Antichità di Sulaymaniyah e Garmian, conduce attualmente due principali progetti di ricerca, il primo focalizzato sullo studio del monumento sasanide di Paikuli e il secondo dedicato allo studio e alla valorizzazione della Cittadella di Erbil ${ }^{4}$.

Luca Colliva e Serenella Mancini

\footnotetext{
$1 \quad$ I paragrafi 1 e 7 sono stati scritti da entrambi gli autori; i paragrafi 2-4 dal Dr. Luca Colliva e i paragrafi 5-6 dalla Dr.ssa Serenella Mancini.

2 Tutte le attività di MAIKI sono realizzate grazie al contributo del Ministero degli Affari Esteri italiano e della Cooperazione Italiana allo Sviluppo e al continuo sostegno dell'Ambasciata italiana a Baghdad e del Consolato italiano di Erbil. La MAIKI ringrazia anche 1'Associazione Internazionale di Studi sul Mediterraneo e l'Oriente (ISMEO) per il fondamentale cofinanziamento e BraDypUS. Communicating Cultural Heritage a.s. e BABUINO Unlimited S.R.L. per le preziose sponsorizzazioni date alla missione.

3 Cereti - Giunta 2012; Cereti et al. 2013; Bizzarro - Colliva 2015; Cereti - Colliva 2016.

4 Cereti et al. 2012; Kamal et al. 2014; Bogdani et al. 2015; 2016; Colliva - Terribili 2017; Bogdani - Colliva 2019; Cereti et al. 2019.
}

ISSN 0393-0300; 2724-587X

e-ISSN 2532-5159

doi: 10.53131/VO2724-587X2021_7 


\section{LA CitTAdELla Di ERBIL E LE ATTIVITÀ CONGIUNTE HCECR E MAIKI}

La Cittadella di Erbil, con i suoi 15 ettari di estensione e i suoi circa 30 metri di altezza, è il nucleo fisico e simbolico della moderna capitale della regione autonoma del Kurdistan Iracheno (KRG) ed è riconosciuta come uno dei più importanti siti archeologici della regione (fig. 1) $)^{5}$. Non è un caso che dal 2014 la Cittadella di Erbil sia stata inserita nella lista del Patrimonio Mondiale dell'UNESCO.

Le strutture architettoniche che oggi dominano la sommità del tell sono in gran parte databili al tardo periodo ottomano. Molti di questi edifici sono splendidi esempi dell'architettura di quel periodo, ma, nonostante ciò, la loro importanza, almeno da un punto di vista prettamente archeologico, è poca cosa rispetto a quanto ancora sepolto negli oltre venti metri di stratigrafia archeologica. Il sito oggi conosciuto come Cittadella di Erbil (Erbil Citadel) è stato identificato con l'antica Urbilum dei testi cuneiformi, con l'Arbairā delle iscrizioni in Antico Persiano e con l'Arbēla delle fonti greche. L'esistenza di un importante centro urbano - peraltro abitato in maniera sostanzialmente continuativa fino al secolo scorso - è, inoltre, riportata nelle fonti cuneiformi dal III millennio a.C. ${ }^{6}$ Una continuità insediativa di almeno seimila anni, se confermata dagli studi in corso e dagli auspicabili nuovi scavi archeologici, potrebbe fornire dati essenziali per la comprensione dello sviluppo antropico della regione.

La MAIKI collabora fin dalla sua nascita con l'HCECR e gli attuali progetti comprendono una serie di indagini geofisiche, finalizzate alla comprensione della stratigrafia archeologica del sito, e lo studio congiunto dei materiali ceramici rinvenuti durante gli scavi stratigrafici realizzati tra il 2013 e il 2015 dall'HCECR.

\subsection{Lo studio del materiale ceramico dallo scavo della Cittadella di Erbil condotto dall' HCECR}

Nel 2015 è stato firmato un accordo di collaborazione fra MAIKI e HCECR per lo studio congiunto del corpus ceramico rinvenuto durante lo scavo che ha interessato due distinti settori della Cittadella: l'Area E, collocata a ovest dell'ingresso nord (North Gate), e l'Area G, situata a est dell'ingresso sud (Main Gate) (fig.1a).

Scopo principale del progetto è la catalogazione sistematica del materiale ceramico, l'indagine e la comprensione delle problematiche relazioni di quest'ultimo, che conta più di 10.000 frammenti, con la stratigrafia archeologica.

Durante cinque campagne finora effettuate tutto il materiale ceramico è stato visionato e immagazzinato secondo criteri stratigrafici, per poi concentrarsi sullo studio, la catalogazione, la raccolta di documentazione grafica e fotografica e la registrazione in un apposito database relazionale di circa 1.700 frammenti considerati diagnostici. Sfortunatamente la cancellazione di alcune campagne di studio, dovuta prima alle problematiche seguite al referendum sull'indipendenza del 2017 e, più recentemente, alla pandemia globale, non ha ancora permesso di ultimare lo studio e l'analisi dei dati raccolti. I risultati preliminari, tuttavia, sono molto significativi e meritano un'attenta valutazione.

Curtis 2005, 175, 189; Al Yaqoobi et al. 2015; Al Yaqoobi et al. 2016b.

6 Unger 1929; Sourdel 1978; Curtis 2005; Novácek 2009; Hansman 2011; MacGinnis 2014; Cereti et al. 2013; Al Yaqoobi et al. 2016b. 
Lo studio sistematico del materiale ceramico ha permesso di individuare produzioni ceramiche attribuibili a un ampio arco cronologico che si estende almeno dal periodo ellenistico a quello ottomano.

Il materiale identificato include alcune produzioni provenienti dall'altopiano iranico e dall'area siriana che forniscono preziosi dati sui contatti commerciali che questa regione aveva durante il periodo islamico e confermano il ruolo nevralgico di questo sito.

Al progetto hanno preso parte: come MAIKI, oltre a chi scrive, Marco Galuppi e la Dott.ssa Aila Santi; come HCECR, Sangar Mohammed Abdullah, Chinar Faris Mohammed e Hezha Zayar Mohammed ${ }^{7}$.

È importante sottolineare che tutti i dati archeologici e stratigrafici sono stati forniti dall'HCECR, che ha effettuato lo scavo con la consulenza del Dr. John MacGinnis e della Dr.ssa Mary Shepperson ${ }^{8}$. Non rientra tra gli obiettivi del nostro progetto la revisione puntuale della stratigrafia identificata durante lo scavo; i nostri studi si sono invece focalizzati sull'implementazione della periodizzazione esistente attraverso l'analisi comparata dei dati stratigrafici e di quelli ottenuti dallo studio del materiale ceramico. I dati forniti dallo studio delle produzioni identificate e dal loro inserimento in un corretto contesto archeologico, infatti, hanno permesso a chi scrive di rivedere la periodizzazione della stratigrafia rinvenuta durante lo scavo e di presentare nuove proposte per la datazione delle diverse fasi identificate.

\section{L'AREA E}

Nell'area E è stata indagata un'ampia porzione del muro perimetrale della Cittadella e alcuni ambienti interni localizzati a ridosso del muro. La sezione di muro perimetrale che è stata investigata include una torre a pianta circolare e mostra chiari segni di ricostruzioni o integrazioni che identificano diverse fasi costruttive. Lo scavo di questo settore ha visto l'apertura di una trincea principale $(20 \times 15 \mathrm{~m})$ e di una trincea secondaria $(6 \times 8 \mathrm{~m})$ in prossimità dell'angolo nord-ovest della precedente (fig. 2). La stratigrafia investigata ha raggiunto una profondità di circa otto metri e lo studio comparato dei dati stratigrafici e dei materiali ha permesso a chi scrive di identificare nove distinte fasi archeologiche ${ }^{9}$.

Alla Fase 9 appartengono solo due muri in mattoni crudi (SSU 155 e SU 164) ${ }^{10}$ che corrispondono ai più antichi resti del muro perimetrale della Cittadella fino a ora identificati. Nessun frammento ceramico è associato a queste unità stratigrafiche e non abbiamo dati per

Gli autori e tutto il gruppo di ricerca ringraziano il direttore della MAIKI, Prof. Carlo G. Cereti, l'Head of the HCECR, Nihad Qoja, il precedente Head of the HCECR, Dr. Dara al Yaqoobi, e la Erbil Citadel Site Manager, arch. Ranan Khasraw Tawfiq, per il continuo e indispensabile supporto dato a questo progetto.

8 Al Yaqoobi et al. 2016a; Al Yaqoobi et al. 2018

9 Le nove fasi identificate durante questo studio costituiscono un'implementazione delle tre "major phases of fortification walls" identificate durante lo scavo (Al Yaqoobi et al. 2018, 451-455). Diversamente da quanto proposto nei rapporti di scavo pubblicati fino a ora, nella sequenza da noi proposta le fasi hanno una numerazione crescente a partire dalla più recente.

10 La sigla SU (Stratigraphic Unit) indica gli strati di accumulo; la sigla SSU (Structural Stratigraphic Unit) identifica le unità stratigrafiche strutturali, mentre la sigla NSU (Negative Stratigraphic Unit) caratterizza le unità stratigrafiche negative. Le unità stratigrafiche negative (NSU), non incluse nelle sequenze stratigrafiche originali di Area E e di Area G, sono state aggiunte da chi scrive, sulla base dell'osservazione diretta della stratigrafia ancora visibile, al fine di consentire una più dettagliata articolazione della sequenza stratigrafica implementata. 
l'attribuzione cronologica di questa fase ${ }^{11}$.

La Fase 8 sembra corrispondere a una riedificazione o riparazione del muro perimetrale, le cui tracce sono visibili nell'estensione nord-occidentale dello scavo. Solo un frammento ceramico diagnostico è stato ritrovato negli strati di Fase 8, ma la sua attribuzione al periodo islamico suggerisce che provenga da un diverso contesto archeologico. Ipotizziamo che la presenza di questo frammento in SU 161 sia probabilmente da collegare a un cavo di fondazione (o una buca), scavato in occasione delle attività costruttive caratterizzanti alcune delle fasi successive, ma non identificato durante lo scavo stratigrafico (tab. 1, Fase 8).

Il materiale ceramico proveniente dalle unità stratigrafiche di Fase 7 suggerisce, nel suo complesso, una datazione al periodo partico o sasanide. Le analisi al C14 effettuate su campioni provenienti da SU 141-153 - uno strato di cenere che si estende su tutta l'area esterna al muro perimetrale - hanno fornito una datazione al I-II secolo d.C. ${ }^{12}$ Tuttavia, la presenza di frammenti riconducibili, non senza dubbi, al periodo sasanide ${ }^{13}$ e il numero crescente di frammenti di ceramica invetriata monocroma turchese, anche opaca, e di frammenti databili al periodo islamico ${ }^{14}$, suggeriscono una datazione più tarda. In considerazione di ciò, è forse possibile interpretare lo strato di cenere SU 141-153 come un'azione di livellamento che ha movimentato materiale appartenente a periodi precedenti o come i resti di un incendio che ha bruciato materiale ligneo appartenente a strutture più antiche.

La presenza di frammenti verosimilmente attribuibili, in particolare sulla base dei corpi ceramici, a produzioni dell'età del bronzo e del periodo Neo-assiro, come preliminarmente suggerito anche dal Dott. Mustafa Ahmad, che per primo ha visionato il materiale ceramico subito dopo lo scavo ${ }^{15}$, conferma l'esistenza di una stratigrafia archeologica più antica, ma sembra essere ricollegabile, almeno in questi contesti, a materiale in deposizione secondaria e non fornisce dati utili alla datazione di queste fasi.

La datazione proposta per la Fase 6 sembra evidenziare una significativa lacuna della stratigrafia, soprattutto se la prosecuzione degli studi dovesse confermare, per la Fase 7, nonostante la già evidenziata presenza di materiale di periodo islamico, una cronologia non successiva al periodo sasanide. La presenza di produzioni come monochrome stonepaste ware, sgraffiato monochrome ware e moulded relief ware (tab. 1, Fase 6), suggerisce, infatti, per la Fase 6, una datazione tra il XII e il XIII secolo d.C.

11 La dimensione dei mattoni crudi (circa $40 \times 40 \mathrm{~cm}$ ) e i confronti con le attestazioni in ambito mesopotamico hanno suggerito agli archeologi che hanno effettuato lo scavo, non senza molte incertezze, una datazione al III millennio a.C. (Al Yaqoobi et al. 2016a, 8) o più precisamente al periodo Neo-Assiro (Al Yaqoobi et al. 2018, 451-452). I materiali ceramici delle fasi successive, ascrivibili a un arco cronologico non precedente al periodo partico-sasanide, sembrano suggerire, invece, una datazione più tarda. Le prove su cui si basa la possibile datazione al II-I millennio a.C. appaiono ancora più incerte se si considera che mattoni in argilla cruda con dimensioni simili sono attestati, se si accetta di prendere in considerazione confronti geograficamente così distanti, anche a Nisa, in edifici databili al periodo partico (Lippolis 2008, 217, nota 5, e relativa bibliografia).Non possiamo non notare che una datazione al periodo partico delle strutture di Fase 9 sarebbe più coerente con il materiale ceramico rinvenuto nelle fasi seguenti.

12 Al Yaqoobi et al. 2018, 452.

13 La mancanza di forme o decorazioni chiaramente datanti ci porta a considerare queste datazioni incerte.

14 Anche in questo caso la presenza in queste unità stratigrafiche di materiali provenienti da contesti archeologici più recenti sembra essere connessa alla presenza di fosse o buche non identificate durante lo scavo.

15 Comunicazioni personali; si veda anche Al Yaqoobi et al. 2018, 452. 
Non sono stati individuati strati chiaramente connessi con il primo periodo islamico e non si può escludere che questi strati siano stati parzialmente o completamente rimossi, almeno in questo settore, da possibili azioni di livellamento connesse con le successive fasi costruttive.

La Fase 5 è caratterizzata dalla realizzazione di alcune strutture minori (tab. 1, Fase 5) addossate alle strutture del muro perimetrale e non è stato rinvenuto materiale ceramico utile alla datazione di queste strutture.

La Fase 4 e la Fase 3 sono, al contrario, molto ricche di materiali, per la maggior parte in deposizione secondaria. La datazione di queste produzioni, in particolare champlevé e lustre ware, suggerisce un terminus post quem tra la fine del XII e il XIV secolo d.C. (tab. 1, Fase 4, Fase 3).

Lo studio delle fasi più recenti, Fase 2 e Fase 1, mostra nuovamente diversi materiali chiaramente in deposizione secondaria. Ancora una volta il materiale ceramico può fornire solo un terminus post quem, ma i dati raccolti suggeriscono per la Fase 2 una possibile datazione al XIII-XIV secolo d.C. (tab. 1, Fase 2).

Non è stata identificata nessuna fase chiaramente databile al XV-XVII secolo d.C. A questo riguardo, non si esclude che la prosecuzione degli studi possa portare a una variazione delle datazioni e della suddivisione e interpretazione stratigrafica delle Fasi 1 e 2 o che, ancora una volta, azioni di livellamento, connesse alle attività di ricostruzione del muro perimetrale, abbiano rimosso parte della stratigrafia archeologica.

Per la Fase 1, equivalente all'ultima parte della "Phase 3" identificata dagli archeologi che hanno realizzato lo scavo, i materiali ceramici studiati sembrano confermare la datazione al XVIII secolo d.C. già proposta nei rapporti di scavo ${ }^{16}$. Questa datazione è confermata anche dal ritrovamento di diverse pipe ottomane, Ottoman clay pipes ${ }^{17}$. Lo studio comparato dei materiali ceramici e dei dati stratigrafici e, ad esempio, il ritrovamento di colour splashed ware (IX-X secolo d.C.) insieme a moulded relief ware (XII-XIII secolo d.C.) e Ottoman clay pipes (SU 002, SU 057 e SU 105); Sasanian stamped ware insieme a colour splashed ware e moulded relief ware (SU 084) e Raqqa ware (XII-XIII secolo d.C.), moulded relief ware (XII-XIII secolo d.C.) e sgraffiato splashed ware (XII-XIV secolo d.C.) insieme a Ottoman painted ware (SU 103), confermano la deposizione secondaria della maggior parte dei materiali archeologici e le significative attività di livellamento già ipotizzate nei rapporti di scavo (tab. 1, Fase 1).

\section{L'AREA G}

Nell'area $\mathrm{G}$ è stata investigata una piccola porzione del muro perimetrale della Cittadella (fig. 3), parzialmente esposta durante i lavori di costruzione della porta monumentale realizzata negli anni ' 70 del secolo scorso. Lo studio del materiale ceramico e il confronto con i dati stratigrafici hanno portato chi scrive all'identificazione di quattro fasi archeologiche:

La Fase 4 e la Fase 3 mostrano due distinti momenti della costruzione del muro perimetrale della Cittadella. Sono state identificate tre unità stratigrafiche strutturali (SSU

$16 \quad$ Al Yaqoobi et al. 2018, 453-454.

17 Uno studio dettagliato delle Ottoman clay pipes ritrovate sulla Cittadella di Erbil, condotto dalla Dr.ssa Serenella Mancini, è attualmente in corso di pubblicazione. 
206 e SSU 209 per Fase 4 e SSU 204 per Fase 3), ma nessuno degli strati di accumulo ad esse legate è stato scavato e non è stato ritrovato materiale archeologico che possa fornire indicazioni utili alla datazione di queste due fasi (tab. 2, Fase 4 e Fase 3).

La Fase 2 include due strati di accumulo (SU 207 e SU 208), addossati alle strutture dei periodi precedenti, forse con lo scopo di rafforzare il muro perimetrale della Cittadella. La natura dei due strati e l'insieme del materiale rinvenuto suggeriscono una deposizione secondaria del materiale ceramico la cui datazione può quindi fornire solo un terminus post quem per l'inquadramento cronologico della fase. I frammenti ceramici identificati, in particolare un frammento di sgraffiato splashed ware e un frammento di moulded relief ware (SU 208), inducono a proporre, per questa fase, una datazione non anteriore al XII-XIII secolo d.C. (tab. 2, Fase 2).

La Fase 1 consiste in una serie di strati di accumulo (SU 200, SU 201, SU 202, SU 203 e SU 205) che si appoggiano agli strati di Fase 2. Anche per la Fase 1 i contesti suggeriscono una deposizione secondaria della ceramica e un conseguente terminus post quem per la datazione di questa fase. Il vasellame identificato, in particolare lustre ware, moulded relief ware, sgraffiato splashed ware e sgraffiato monochrome ware, suggerisce, per questa fase, una datazione non anteriore al XII-XIV secolo d.C. (tab. 2, Fase 1).

Luca Colliva

\section{LA METODOLOGIA DI STUDIO APPLICATA AL CORPUS CERAMICO DELLA CITTADELLA}

Il corpus ceramico attestato sulla Cittadella di Erbil è consistente ed eterogeneo. Le due classi della ceramica non invetriata e invetriata presentano infatti una vasta gamma di produzioni relative a un ampio arco cronologico dal periodo ellenistico fino al periodo ottomano.

In considerazione di queste caratteristiche è stato necessario adottare un sistema di classificazione che fosse duttile e applicabile a qualsiasi tipologia ceramica, si sono infatti presi in considerazione tutti gli aspetti tassonomici, dimensionali e qualitativi al fine di ottenere una descrizione il più possibile completa dei frammenti studiati ${ }^{18}$. La metodologia di studio dei materiali ceramici è infatti finalizzata alla classificazione e all'identificazione delle diverse produzioni attestate, alla comprensione del livello tecnologico di queste ultime e all'individuazione di eventuali produzioni locali e manufatti di importazione.

Si è scelto di limitare lo studio, almeno in questa prima fase, al solo materiale ceramico considerato diagnostico. Sono stati quindi selezionati tutti i frammenti che per forma e/o particolarità tecnologiche possono considerarsi significativi per l'identificazione delle produzioni ceramiche attestate nel sito.

18 Questa metodologia è stata messa a punto dalla Dr.ssa A. Fusaro, da M. Galuppi e dalla Dr.ssa S. Mancini per lo studio preliminare del materiale ceramico rinvenuto durante le attività di ricognizione della MAIKI connesse con la realizzazione di una carta archeologica dell'area in prossimità del sito di Paikuli, tra i fiumi Basra e Dyala (Kamal et al. 2014, 29-30; Bogdani - Colliva 2019, 55-62), e successivamente implementata da chi scrive e da M. Galuppi per lo studio in oggetto. 


\subsection{La classificazione del materiale ceramico}

Nel processo di classificazione sono stati considerati diversi indicatori ritenuti determinanti al fine di ottenere una descrizione accurata e completa dei frammenti presi in esame.

Il primo indicatore, discriminante fondamentale nel processo di analisi, è il corpo ceramico, di cui vengono analizzati il colore, la porosità ${ }^{19}$, la durezza, la compattezza e la presenza o meno di inclusi vegetali e minerali ${ }^{20}$.

Il secondo indicatore preso in esame è la tecnica di modellazione.

Il terzo indicatore è costituito dagli eventuali trattamenti superficiali e/o rivestimenti (argillosi e/o vetrosi). Per quanto concerne i rivestimenti argillosi, se ne descrive la consistenza (ingobbio o wash) e il colore; per i rivestimenti vetrosi l'aspetto (vetrina trasparente o vetrina opaca) e il colore. Quando presenti si procede, inoltre, alla descrizione di eventuali alterazioni, sia primarie che secondarie ${ }^{21}$.

Le tecniche decorative sono il quarto indicatore preso in considerazione e possono riscontrarsi sia singolarmente che in associazione fra loro. Sebbene lo studio sia ancora in una fase preliminare, è stato possibile riconoscere l'impiego di un'ampia gamma di tecniche decorative: dalle più semplici, come l'incisione, l'excisione o la decorazione applicata, alle più complesse, come la decorazione dipinta a lustro metallico.

Segue infine, quando possibile, uno studio morfologico del frammento per tentare di identificarne la forma originaria.

\section{Il CORPUS CERAMICO DELla CITTADELla DI ERBIL E ALCUNE DELLE PRINCIPALI} PRODUZIONI CERAMICHE DI PERIODO ISLAMICO

Il corpus ceramico proveniente dagli scavi della Cittadella di Erbil è consistente ed estremamente vario. Finora sono stati visionati circa 10.000 frammenti, il $17 \%$ dei quali è stato riconosciuto come diagnostico.

Il materiale ceramico attribuibile al periodo islamico è il più consistente e copre un arco cronologico notevolmente ampio che sembra compreso almeno fra il IX e il XIX secolo.

Benché lo studio del materiale sia ancora in corso, verranno presentate alcune delle principali produzioni ceramiche di periodo islamico.

\subsection{Classe della ceramica non invetriata}

La ceramica non invetriata costituisce circa il 68\% dell'intero corpus attribuibile al periodo islamico. Sono attestati principalmente unglazed plain ware, unglazed ware with incised and carved decoration e unglazed ware with applied decoration. Da un punto di vista morfologico sono perlopiù presenti bacini, brocche e giare di medie dimensioni,

19 La porosità è determinata dalla presenza di vacuoli di cui si indica quantità, dimensione e forma.

20 Nell'impossibilità di valutare l'effettiva natura mineralogica degli inclusi attraverso l'osservazione macroscopica, l'esposizione è descrittiva e non interpretativa. Per ogni tipo di incluso identificato si segnalano il colore, l'aspetto (brillantezza, opacità o trasparenza), la quantità, la distribuzione, la forma e infine le dimensioni.

21 Per alterazioni primarie si intendono tutte quelle imperfezioni che possono essere direttamente legate ad azioni effettuate durante la produzione dell'oggetto. Le alterazioni secondarie sono provocate da danni o depostiti sull'oggetto connessi ad eventi successivi alla fase di produzione e di probabile uso dell'oggetto, come quelli causati, per esempio, dalla giacitura prolungata nel terreno. 
principalmente prodotti con corpi ceramici mediamente grossolani.

Finora la frammentarietà, lo stato di conservazione, la natura dei contesti archeologici in cui è stata rinvenuta la maggior parte del materiale ceramico della Cittadella e l'assenza di confronti significativi con materiale proveniente da contesti datati e appartenenti a siti archeologici della Mesopotamia settentrionale non hanno permesso una precisa attribuzione cronologica per queste produzioni.

\subsubsection{Unglazed relief ware}

Dodici frammenti appartenenti a giare di grandi dimensioni si sono rivelati di particolare interesse per la notevole ricchezza decorativa che li caratterizza. Le decorazioni sono realizzate con molteplici tecniche ma le più attestate sono la decorazione applicata, modellata e incisa ${ }^{22}$. Non è nota l'area di produzione dell'unglazed relief ware, tuttavia si suppone che possa essere collocata nella valle dell'Eufrate, nel nord della Siria e nel nord dell'Iraq. L'attribuzione cronologica di questa produzione risale al XII-XIII secolo d.C. ${ }^{23}$

\subsubsection{Moulded relief ware}

È stato identificato un gruppo di circa 90 frammenti riconducibili al moulded relief ware.

Il materiale ceramico appartenente a questa produzione è stato chiaramente realizzato con la caratteristica tecnica di modellazione a matrice. Sulla superficie interna di alcuni di questi frammenti, infatti, sono stati trovati sia un cordolo di argilla, solitamente riscontrabile in corrispondenza della giuntura delle due metà realizzate con le matrici, sia tracce di lavorazione e rifinitura. La maggior parte di questi frammenti presenta una notevole complessità decorativa che rende evidente l'uso di matrici certamente realizzate da artigiani specializzati.

Tranne che per un esemplare, lo stato frammentario del materiale non ha consentito uno studio morfologico. Tuttavia, si può supporre che i frammenti appartenessero a brocche di medie dimensioni caratterizzate da pareti sottili: lo spessore dei frammenti varia per lo più fra i 3 e i $7 \mathrm{~mm}$.

Per quanto riguarda gli aspetti decorativi, si riscontrano motivi geometrici, vegetali ed epigrafici/pseudo-epigrafici. Questi ultimi sono ben eseguiti, le lettere sono arrotondate e rifinite con cura, soprattutto in considerazione della dimensione del motivo epigrafico, che, di solito, è inferiore a $2 \mathrm{~cm}$ di altezza.

Due esemplari appartenenti a questa produzione sono particolarmente rilevanti: il primo frammento reca la figura di una sfinge con la testa sormontata da una corona; il corpo di felino presenta ali che si diramano sul dorso (fig. 4). La creatura è in piedi su uno sfondo arabescato $^{24}$. Il secondo frammento consiste nella parte superiore del corpo di una brocca, probabilmente ansata, la cui decorazione è costituita da una serie di personaggi maschili stanti (probabilmente musicisti, danzatori o anche coppieri) su uno sfondo arabescato. Le figure sono alternate a medaglioni costituiti da una fascia perlinata contenente un motivo epigrafico che racchiude a sua volta l'immagine stilizzata di un uccello (forse un pavone). È molto

\footnotetext{
Questa produzione è stata identificata e studiata per la prima volta da Reitlinger nel 1951.

Reitlinger 1951; Riis et al. 1957, numeri 844-846.

24 Per un approfondimento si veda Mancini 2020.
} 
verosimile che questo tipo di decorazione, come già ipotizzato da molti studiosi ${ }^{25}$, sia ispirato a oggetti in metallo prezioso prodotti fra il XII e il XIV secolo. Nel complesso tutti i frammenti di moulded relief ware rinvenuti presso la Cittadella di Erbil, proprio per la loro complessità decorativa, possono essere datati a questo stesso periodo e non si esclude, inoltre, che siano stati importati dall'area siro-mesopotamica.

\subsection{Classe della ceramica invetriata}

La classe della ceramica invetriata di periodo islamico costituisce circa il $32 \%$ della selezione del materiale diagnostico. Sono attestate numerose produzioni riconducibili a un ampio arco temporale compreso almeno fra il IX e il XIX secolo d.C.

\subsubsection{Monochrome ware}

Il monochrome ware è la produzione più attestata e copre circa il $25 \%$ della classe invetriata. I frammenti presentano un'invetriatura trasparente principalmente verde o turchese. È attestato anche l'impiego di un'invetriatura di colore giallo, sebbene in minori quantità. L'invetriatura verde è spesso applicata sopra un ingobbio bianco ben visibile in frattura.

Dal punto di vista morfologico sono presenti soprattutto forme aperte: la forma più attestata è una ciotola con orlo arrotondato e base ad anello.

L'attribuzione cronologica del monochrome ware rappresenta una problematica tutt'ora irrisolta. Questa produzione è una delle più diffuse territorialmente e copre, inoltre, un ampio arco temporale dal periodo partico al periodo islamico. La continuità non solo tecnologica, ma, talvolta, anche morfologica, complica ulteriormente l'identificazione cronologica di questa produzione, che è ancora in fase di studio.

\subsubsection{Colour splashed ware}

Sono stati identificati 43 frammenti ceramici appartenenti al colour splashed ware, principalmente prodotti con un corpo ceramico mediamente compatto, di colore marrone chiaro e caratterizzato dalla presenza di inclusi bianchi opachi di piccole dimensioni.

Tutti i frammenti presentano, sotto la vetrina trasparente incolore, l'applicazione di uno spesso ingobbio bianco. Gli splashes sono principalmente bicromi (verdi e gialli), talvolta è attestata la policromia con l'aggiunta di splashes di colore bruno.

Dal punto di vista morfologico sono state identificate principalmente forme aperte con una sola eccezione: un frammento di collo che conserva l'orlo e l'attacco di un'ansa a nastro verticale probabilmente appartenente a una piccola brocca. Nella maggior parte dei casi è stato impossibile effettuare uno studio morfologico a causa delle dimensioni ridotte dei frammenti. Questa produzione è datata al IX-X secolo d.C. ${ }^{26}$

Baer 1989; Mancini 2020, 271.

26 La produzione è stata introdotta in un secondo momento rispetto alle produzioni più note del cosiddetto "Samarra Horizon", probabilmente durante la prima metà del IX secolo (Northedge - Kennet 1994, 33). Si veda anche Priestman 2021, 106-108. 


\subsubsection{Sgraffiato monochrome ware e sgraffiato splashed ware}

Nel corpus sono presenti frammenti attribuibili allo sgraffiato monochrome ware e allo sgraffiato splashed ware, che costituiscono circa l' $8 \%$ della classe invetriata. Per quanto concerne lo sgraffiato monochrome ware, la vetrina monocroma verde trasparente è la più attestata, ma sono presenti anche frammenti con invetriatura trasparente gialla. Lo sgraffiato splashed ware ha un'invetriatura trasparente incolore e una decorazione splashed policroma, principalmente verde e gialla. Entrambi i ware sono stati prodotti con un corpo ceramico di colore marrone chiaro molto compatto. Sono attestate esclusivamente forme aperte, principalmente ciotole con orlo sottile leggermente estroflesso e basi ad anello. I motivi decorativi sono molto semplici, solitamente composti da incisioni curvilinee e spirali. La datazione ipotizzata per questo materiale parte dal XII-XIII secolo fino al XIV secolo d.C., momento in cui queste produzioni sembrano cessare completamente in tutto l'Iraq, l'Iran e l'Asia centrale ${ }^{27}$.

\subsubsection{Lustre ware}

Sono stati rinvenuti undici frammenti decorati con la tecnica della pittura a lustro metallico e prodotti con corpo ceramico artificiale (stonepaste) ${ }^{28}$. I frammenti hanno caratteristiche tecnologiche diverse. Alcuni frammenti sono stati prodotti con un corpo ceramico artificiale estremamente compatto, caratterizzato da un colore bianco. La maggior parte di questi frammenti è rivestita di una spessa invetriatura bianca opaca, di notevole qualità e perfettamente conservata, e presenta una decorazione dipinta monocroma a lustro metallico di colore giallo-oro. In un solo frammento si riscontra l'associazione della pittura a lustro metallico con una pittura monocroma in blu cobalto sotto vetrina. Purtroppo, le ridotte dimensioni dei frammenti non hanno consentito uno studio morfologico.

Un secondo gruppo di sei frammenti è presumibilmente attribuibile all'area siriana. Questo gruppo è caratterizzato da un corpo ceramico artificiale molto poroso di colore rosa tendente al grigio. Anche in questo caso la pittura a lustro è monocroma e si abbina a una decorazione dipinta in blu cobalto sotto vetrina. Ancora una volta è stato impossibile procedere a uno studio morfologico, ma la maggior parte dei frammenti sembra essere riconducibile a forme aperte. In un solo caso, P01238, la forma è verosimilmente chiusa, si tratta di una parete con corpo ceramico artificiale estremamente poroso e decorazione dipinta monocroma a lustro metallico su un'invetriatura blu. Questo frammento è probabilmente databile al XII-XIII secolo d.C. ${ }^{29}$

Tutti gli esemplari di lustre ware provenienti dall'area iranica e siriana vennero importati ad Erbil, con ogni probabilità, a partire dalla seconda metà del XII secolo sino all'inizio del XIV d.C.

27 Kennet 2004; Nováček 2009.

28 Il corpo ceramico artificiale è stato denominato nella letteratura scientifica in diversi modi: stonepaste, quartzfrit-clay paste, quartz-frit, fritware, faience, impasto artificiale (artificial paste), impasto siliceo (siliceous paste), $k \bar{a} s h \bar{\imath}$ (Rugiadi 2011;2016). Per questo studio si è scelto di utilizzare il termine stonepaste.

29 Lane 1957, 15-17; Priestman 2021, 135-136. 


\subsubsection{Raqqa ware}

Un'altra produzione rinvenuta nella Cittadella di Erbil è il così detto Raqqa ware, attestata, per il momento, da un solo frammento. Quest'ultimo è stato prodotto con un corpo ceramico artificiale estremamente poroso di colore rosso-grigio e presenta una decorazione dipinta monocroma nera sotto vetrina turchese. Il frammento, di dimensioni ridotte, appartiene probabilmente a una forma chiusa e può essere datato al XII-XIII secolo d.C. ${ }^{30}$

\subsubsection{Underglazed black painted ware}

Particolarmente interessante è un gruppo di otto frammenti che presentano la stessa tecnica decorativa del frammento di Raqqa ware, una decorazione dipinta monocroma nera sotto vetrina turchese, che, però, è applicata su oggetti realizzati con un corpo ceramico argilloso e non artificiale. Il corpo ceramico, di colore marrone, è caratterizzato da una porosità media e dalla presenza di inclusi minerali, di piccole dimensioni, di colore rosso e bianco opaco. I motivi decorativi attestati su questi otto frammenti sono anch'essi simili a quelli che ritroviamo nel più raffinato Raqqa ware di origine siriana e questo conferma l'ipotesi che questa produzione ne sia un'imitazione (fig. 5). La provenienza di questa produzione, che viene in questa sede definita underglazed black painted ware, è ancora incerta: oggetti simili sono stati rinvenuti in diversi contesti di area iranica e siriana e coprono un ampio arco cronologico (XIV- XVII secolo d.C. o successivo) ${ }^{31}$.

\subsection{Indicatori di produzione locale}

Sono stati identificati tre oggetti che possono essere riconducibili a un contesto produttivo: due distanziatori (trivet; SU 002 e SU 101), che presentano entrambi tracce di vetrina (fig. 6a), e una barra di fornace (kiln peg; SU 029; fig. 6b), tutti rivenuti nei contesti stratigrafici di Fase 1 e Fase 2 (tab. 1, Fase 1, Fase 2). Questi elementi suggeriscono chiaramente la possibilità di una produzione di materiale ceramico sulla Cittadella di Erbil e la presenza di tracce di vetrina sui distanziatori sembra indicare, inoltre, una produzione locale di materiale invetriato. Non è stato possibile, purtroppo, fornire una precisa attribuzione cronologica a questa attività produttiva in quanto il materiale presente negli strati di rinvenimento è in deposizione palesemente secondaria.

Serenella Mancini

\section{CONCLUSIONI}

Lo studio effettuato finora sul corpus ceramico proveniente dagli scavi condotti dall'HCECR sulla Cittadella di Erbil ha permesso di individuare diverse produzioni significative, attribuibili a un ampio arco cronologico che si estende almeno dal periodo ellenistico a quello ottomano. Il corpus databile al periodo islamico è sicuramente il più consistente ed evidenzia una panoramica produttiva estremamente variegata, caratterizzata in gran parte dalla presenza di materiale importato dalla Siria e dall'altopiano iranico.

La maggior parte delle unità stratigrafiche identificate durante lo scavo del Muro Perimetrale della Cittadella nelle Aree E e G è stata probabilmente originata da attività di

30 Porter 1981; Jenkins-Madina 2016

31 Priestman 2021, 147-148. 
livellamento connesse alle diverse fasi costruttive della struttura. La natura di questi contesti archeologici è confermata anche dallo studio comparato del materiale ceramico che, nella maggior parte dei casi, è stato rinvenuto in contesti di deposizione secondaria e che, di conseguenza, può fornire solo dati utili a identificare un terminus post quem per la datazione dei contesti archeologici di rinvenimento.

Nonostante le problematiche evidenziate e lo stadio preliminare dei dati raccolti, lo studio comparato del materiale ceramico, realizzato dal gruppo di ricerca dell'HCECR e della MAIKI, ha permesso il riconoscimento di alcune significative produzioni ceramiche e ha fornito dati fondamentali per una più dettagliata articolazione della stratigrafia archeologica e per la datazione delle diverse fasi identificate (tabb. 1 e 2).

Le principali produzioni ceramiche importate sono sicuramente oggetti di lusso come il frammento di Raqqa ware e i frammenti di lustre ware provenienti dall'area siriana e iranica. Per quanto concerne la classe non invetriata sembra plausibile l'importazione di oggetti attribuibili all'unglazed relief ware e al moulded relief ware che mostrano notevoli correlazioni con oggetti appartenenti alle stesse produzioni e rinvenuti nell'area mesopotamica

La presenza di materiale ceramico proveniente dalle aree siriana e mesopotamica e dall'altopiano iranico non solo conferma l'importanza del sito, ma prova l'esistenza di una rete commerciale che, almeno a partire dal periodo islamico, connetteva Erbil con le regioni confinanti.

La presenza di due trivet, usati come distanziatori durante la cottura del vasellame, e di un kiln peg, in Fase 1 e Fase 2 (tab. 1, Fase 1, Fase 2; fig. 6), suggerisce, inoltre, l'esistenza sulla Cittadella di fornaci per la produzione di ceramica, ma, sfortunatamente, la natura degli strati in cui questi oggetti sono stati rinvenuti non fornisce dati utili per stabilire l'entità di questa produzione o per datare con precisione l'inizio e la durata di questa attività.

Allo stato attuale la letteratura scientifica non fornisce ancora un panorama esaustivo delle produzioni ceramiche di questa regione, ma la quantità e qualità dei materiali ceramici rinvenuti, le produzioni attestate, la probabile presenza di una produzione locale e i collegamenti commerciali, provati dai numerosi rinvenimenti, confermano il ruolo chiave della Cittadella di Erbil. Questo sito, infatti, dimostra di avere il potenziale per diventare uno dei siti guida per lo studio della produzione ceramica della regione. In ragione di ciò il progetto di studio e analisi del corpus ceramico della Cittadella di Erbil, effettuato dall'HCECR e dalla MAIKI, può essere considerato un importante punto di partenza e può fornire un apporto considerevole alla comprensione delle dinamiche socio-economiche e commerciali relative a questa regione e ai territori con quest'ultima confinanti.

Luca Colliva e Serenella Mancini 


\begin{tabular}{|c|c|c|c|c|}
\hline \multicolumn{5}{|c|}{ AREA E - PERIODIZZAZIONE E PRINCIPALI PRODUZIONI CERAMICHE } \\
\hline FASI & $\begin{array}{l}\text { DATAZIONE } \\
\text { PROPOSTA }\end{array}$ & $\begin{array}{c}\text { UNITÀ } \\
\text { STRATIGRAFICA }\end{array}$ & $\begin{array}{l}\text { PRINCIPALI PRODUZIONI } \\
\text { CERAMICHE IDENTIFICATE }\end{array}$ & $\begin{array}{l}\text { DATAZIONE PROPOSTA } \\
\text { PER LE PRODUZIONI } \\
\text { CERAMICHE }\end{array}$ \\
\hline \multirow{10}{*}{1} & \multirow{10}{*}{$\begin{array}{l}\text { PERIODO } \\
\text { OTTOMANO }\end{array}$} & SU 001 & $\begin{array}{c}\text { Moulded relief ware } \\
\text { Ottoman painted ware? } \\
\text { Sgraffiato monochrome ware } \\
\text { Sgraffiato splashed ware }\end{array}$ & $\begin{array}{l}\text { XII-XIII secolo d.C. } \\
\text { Periodo ottomano? } \\
\text { XII-XIV secolo d.C. } \\
\text { XII-XIV secolo d.C. }\end{array}$ \\
\hline & & SU 002 & $\begin{array}{c}\text { Colour splashed ware } \\
\text { Monochrome stonepaste ware } \\
\text { Monochrome green ware } \\
\text { Monochrome turquoise ware } \\
\text { Moulded relief ware } \\
\text { Ottoman clay pipe } \\
\text { Ottoman painted ware? } \\
\text { Sgraffiato splashed ware } \\
\text { Trivet }\end{array}$ & $\begin{array}{c}\text { IX-X secolo d.C. } \\
\text { XII-XIV sec d.C. } \\
- \\
- \\
\text { XII-XIII secolo d.C. } \\
\text { Periodo ottomano } \\
\text { Periodo ottomano } \\
\text { XII-XIV secolo d.C. } \\
\text { - }\end{array}$ \\
\hline & & SU 005 & $\begin{array}{l}\text { Monochrome stonepaste ware } \\
\text { Sgraffiato monochrome ware }\end{array}$ & $\begin{array}{l}\text { XII-XIV secolo d.C. } \\
\text { XII-XIV secolo d.C. }\end{array}$ \\
\hline & & SU 014 & $\begin{array}{c}\text { Monochrome turquoise ware } \\
\text { Moulded relief ware } \\
\text { Ottoman clay pipe } \\
\text { Ottoman painted ware? }\end{array}$ & $\begin{array}{l}\text { - } \\
\text { XII-XIII secolo d.C. } \\
\text { Periodo ottomano } \\
\text { Periodo ottomano? }\end{array}$ \\
\hline & & SU 015 & $\begin{array}{l}\text { Colour splashed ware } \\
\text { Ottoman clay pipe }\end{array}$ & $\begin{array}{l}\text { IX-X secolo d.C. } \\
\text { Periodo ottomano }\end{array}$ \\
\hline & & SU 018 & $\begin{array}{c}\text { Colour splashed ware } \\
\text { Sgraffiato monochrome ware }\end{array}$ & $\begin{array}{l}\text { IX-X secolo d.C. } \\
\text { XII-XIV secolo d.C. }\end{array}$ \\
\hline & & SU 029 & Kiln peg & - \\
\hline & & SU 057 & $\begin{array}{c}\text { Colour splashed ware } \\
\text { Monochrome stonepaste ware } \\
\text { Monochrome opaque ware } \\
\text { Monochrome turquoise ware } \\
\text { Sgraffiato monochrome ware } \\
\text { Moulded relief ware } \\
\text { Ottoman clay pipe } \\
\text { Ottoman painted ware? } \\
\text { Underglazed black painted ware }\end{array}$ & $\begin{array}{c}\text { IX-X secolo d.C. } \\
\text { XII-XIV secolo d.C. } \\
\text { Periodo islamico } \\
\text { - } \\
\text { XII-XIV secolo d.C. } \\
\text { XII-XIII secolo d.C. } \\
\text { Periodo ottomano } \\
\text { Periodo ottomano? } \\
\text { XIV-XVII secolo d.C.? }\end{array}$ \\
\hline & & SU 058 & $\begin{array}{l}\text { Moulded relief ware } \\
\text { Ottoman clay pipe }\end{array}$ & $\begin{array}{l}\text { XII-XIII secolo d.C. } \\
\text { Periodo ottomano }\end{array}$ \\
\hline & & SU 084 & $\begin{array}{l}\text { Colour splashed ware } \\
\text { Moulded relief ware } \\
\text { Sasanian stamped ware }\end{array}$ & $\begin{array}{l}\text { IX-X secolo d.C. } \\
\text { XII-XIII secolo d.C } \\
\text { Periodo sasanide }\end{array}$ \\
\hline
\end{tabular}




\begin{tabular}{|c|c|c|c|c|}
\hline & & SU 088 & $\begin{array}{l}\text { Unglazed relief ware } \\
\text { Moulded relief ware? }\end{array}$ & $\begin{array}{l}\text { XII-XIII secolo d.C. } \\
\text { XII-XIII secolo d.C.? }\end{array}$ \\
\hline & & SU 089 & Monochrome turquoise ware & - \\
\hline & & NSU $089 \mathrm{~N}$ & - & \\
\hline & & SU 090 & Moulded relief ware & XII-XIII secolo d.C. \\
\hline & & SU 096 & Monochrome turquoise ware & - \\
\hline & & SU 097 & - & \\
\hline & & SU 098 & - & \\
\hline & & NSU 096N & - & \\
\hline & & NSU $097 \mathrm{~N}$ & - & \\
\hline & & NSU $098 \mathrm{~N}$ & - & \\
\hline & & SU 103 & $\begin{array}{c}\text { Monochrome green ware } \\
\text { Monochrome turquoise ware } \\
\text { Moulded relief ware } \\
\text { Ottoman painted ware? } \\
\text { Raqqa ware } \\
\text { Sgraffiato splashed ware }\end{array}$ & $\begin{array}{c}- \\
- \\
\text { XII-XIII secolo d.C. } \\
\text { Periodo ottomano? } \\
\text { XII-XIII secolo d.C. } \\
\text { XII-XIV secolo d.C. }\end{array}$ \\
\hline & & NSU $103 \mathrm{~N}$ & - & \\
\hline & & SU 105 & $\begin{array}{l}\text { Colour splashed ware } \\
\text { Sgraffiato monochrome ware } \\
\text { Moulded relief ware } \\
\text { Ottoman clay pipe }\end{array}$ & $\begin{array}{l}\text { IX-X secolo d.C. } \\
\text { XII-XIV secolo d.C. } \\
\text { XII-XIII secolo d.C. } \\
\text { Periodo ottomano }\end{array}$ \\
\hline & & SU 099 & $\begin{array}{c}\text { Sgraffiato monochrome ware } \\
\text { Moulded relief ware } \\
\text { Colour splashed ware }\end{array}$ & $\begin{array}{l}\text { XII-XIV secolo d.C. } \\
\text { XII-XIII secolo d.C. } \\
\text { IX-X secolo d.C. }\end{array}$ \\
\hline 2 & $\begin{array}{c}\text { XIII-XIV } \\
\text { SECOLO D.C. } \\
\text { (TERMINUS POST } \\
\text { QUEM). }\end{array}$ & SU 100 & $\begin{array}{c}\text { Colour splashed ware } \\
\text { Fishplate } \\
\text { Monochrome green ware } \\
\text { Monochrome turquoise ware } \\
\text { Moulded relief ware } \\
\text { Sgraffiato monochrome ware } \\
\text { Sgraffiato splashed ware } \\
\text { Unglazed relief ware }\end{array}$ & $\begin{array}{c}\text { IX-X secolo d.C. } \\
\text { IV-I secolo a.C. } \\
- \\
- \\
\text { XII-XIII secolo d.C. } \\
\text { XII-XIV secolo d.C. } \\
\text { XII-XIV secolo d.C. } \\
\text { XII-XIII secolo d.C. }\end{array}$ \\
\hline & & SU 101 & $\begin{array}{c}\text { Moulded relief ware } \\
\text { Sgraffiato splashed ware } \\
\text { Trivet }\end{array}$ & $\begin{array}{l}\text { XII-XIII secolo d.C. } \\
\text { XII-XIV secolo d.C. } \\
-\end{array}$ \\
\hline & & SU107 & & \\
\hline & & SU 109 & & \\
\hline & & SSU 106 & Sgraffiato monochrome ware & XII-XIV secolo d.C. \\
\hline 3 & & SU 115 & $\begin{array}{l}\text { Monochrome ware } \\
\text { Moulded relief ware }\end{array}$ & $\begin{array}{c}- \\
\text { XII-XIII secolo d.C. }\end{array}$ \\
\hline
\end{tabular}




\begin{tabular}{|c|c|c|c|c|}
\hline & \multirow{5}{*}{$\begin{array}{l}\text { XII-XIV SECOLO } \\
\text { D.C. (TERMINUS } \\
\text { POST QUEM). }\end{array}$} & SU 120 & $\begin{array}{c}\text { Colour splashed ware } \\
\text { Monochrome green ware } \\
\text { Monochrome yellow ware } \\
\text { Monochrome stonepaste ware } \\
\text { Moulded relief ware }\end{array}$ & $\begin{array}{c}\text { IX-X secolo d.C. } \\
- \\
- \\
\text { XII-XIV secolo d.C. } \\
\text { XII-XIII secolo d.C. }\end{array}$ \\
\hline & & SSU 094 & Unglazed relief ware & XII-XIII sec d.C. \\
\hline & & SSU 095 & Colour splashed ware & IX-X secolo d.C. \\
\hline & & SU 121 & & \\
\hline & & NSU 094F & & \\
\hline \multirow{9}{*}{4} & \multirow{9}{*}{$\begin{array}{l}\text { XII-XIV SECOLO } \\
\text { D.C. (TERMINUS } \\
\text { POST QUEM). }\end{array}$} & SU 122 & $\begin{array}{c}\text { Monochrome green ware } \\
\text { Monochrome stonepaste ware } \\
\text { Sgraffiato monochrome ware } \\
\text { Moulded relief ware }\end{array}$ & $\begin{array}{l}- \\
\text { XII-XIV secolo d.C. } \\
\text { XII-XIV secolo d.C. } \\
\text { XII-XIII secolo d.C. }\end{array}$ \\
\hline & & SSU 056? & Moulded relief ware & XII-XIII secolo d.C. \\
\hline & & SU 126 & $\begin{array}{l}\text { Sgraffiato monochrome ware } \\
\text { Moulded relief ware } \\
\text { Colour splashed ware }\end{array}$ & $\begin{array}{l}\text { XII-XIV secolo d.C. } \\
\text { XII-XIII secolo d.C. } \\
\text { IX-X secolo d.C. }\end{array}$ \\
\hline & & SU 127 & $\begin{array}{l}\text { Monochrome stonepaste ware } \\
\text { Sgraffiato monochrome ware } \\
\text { Moulded relief ware } \\
\text { Colour splashed ware }\end{array}$ & $\begin{array}{l}\text { XII-XIV secolo d.C. } \\
\text { XII-XIV secolo d.C. } \\
\text { XII-XIII secolo d.C. } \\
\text { IX-X secolo d.C. }\end{array}$ \\
\hline & & SSU 093 & & \\
\hline & & SSU 016 & & \\
\hline & & SSU 123 & $\begin{array}{c}\text { Champlevé } \\
\text { Fishplate } \\
\text { Moulded relief ware }\end{array}$ & $\begin{array}{l}\text { XII-XIII secolo d.C. } \\
\text { IV-I secolo a.C. } \\
\text { XII-XIII secolo d.C. }\end{array}$ \\
\hline & & SU 128 & $\begin{array}{c}\text { Lustre ware } \\
\text { Monochrome stonepaste ware } \\
\text { Moulded relief ware } \\
\text { Sgraffiato splashed ware }\end{array}$ & $\begin{array}{l}\text { XII-XIV secolo d.C. } \\
\text { XII-XIV secolo d.C. } \\
\text { XII-XIII secolo d.C. } \\
\text { XII-XIV secolo d.C. }\end{array}$ \\
\hline & & SU $129-130$ & $\begin{array}{c}\text { Colour splashed ware } \\
\text { Lustre ware } \\
\text { Monochrome stonepaste ware } \\
\text { Moulded relief ware } \\
\text { Unglazed relief ware } \\
\text { Sgraffiato monochrome ware } \\
\text { Sgraffiato splashed ware }\end{array}$ & $\begin{array}{l}\text { IX-X secolo d.C. } \\
\text { XII-XIV secolo d.C. } \\
\text { XII-XIV secolo d.C. } \\
\text { XII-XIII secolo d.C. } \\
\text { XII-XIII secolo d.C. } \\
\text { XII-XIV secolo d.C. } \\
\text { XII-XIV secolo d.C. }\end{array}$ \\
\hline \multirow{4}{*}{5} & \multirow{4}{*}{-} & SSU $018 ?$ & & \\
\hline & & SSU $163 ?$ & & \\
\hline & & SSU 017? & & \\
\hline & & SSU 132 & & \\
\hline
\end{tabular}




\begin{tabular}{|c|c|c|c|c|}
\hline & & SSU 133 & & \\
\hline & & SSU 134 & & \\
\hline & & SSU 137 & & \\
\hline & & SU 135 & & \\
\hline \multirow{6}{*}{6} & \multirow{6}{*}{$\begin{array}{l}\text { XII-XIII SECOLO } \\
\text { D.C. }(\text { TERMINUS } \\
\text { POST QUEM) }\end{array}$} & SSU 119 & Moulded relief ware & XII-XIII secolo d.C. \\
\hline & & SSU 150 & & \\
\hline & & SSU $158 ?$ & & \\
\hline & & NSU 119F & & \\
\hline & & SU 152 & & \\
\hline & & SU 136 & $\begin{array}{l}\text { Monochrome green ware } \\
\text { Monochrome turquoise ware } \\
\text { Monochrome stonepaste ware } \\
\text { Sgraffiato monochrome ware }\end{array}$ & $\begin{array}{c}- \\
- \\
\text { XII-XIV secolo d.C. } \\
\text { XII-XIV secolo d.C. }\end{array}$ \\
\hline \multirow{11}{*}{7} & \multirow{11}{*}{$\begin{array}{c}\text { PERIODO } \\
\text { SASANIADE O } \\
\text { PROTO-ISLAMICO } \\
\text { (presenza di } \\
\text { frammenti di } \\
\text { periodo islamico } \\
\text { fuori contesto?) }\end{array}$} & SU 143 & $\begin{array}{l}\text { Moulded relief ware } \\
\text { Impressed ware? } \\
\text { Fishplate }\end{array}$ & $\begin{array}{l}\text { XII-XIII secolo d.C. } \\
\text { periodo sasanide? } \\
\text { IV-I secolo a.C.? }\end{array}$ \\
\hline & & SU 141 & $\begin{array}{c}\text { Monochrome turquoise ware } \\
\text { Underglazed black painted ware }\end{array}$ & $\begin{array}{c}- \\
\text { XIV-XVII secolo d.C.? }\end{array}$ \\
\hline & & SU 153 & Monochrome turquoise ware & - \\
\hline & & SU 142 & Monochrome stonepaste ware & XII-XIV secolo d.C. \\
\hline & & SU 160 & $\begin{array}{c}\text { Monochrome turquoise ware? } \\
\text { Fishplate }\end{array}$ & $\begin{array}{c}- \\
\text { IV-I secolo a.C.? }\end{array}$ \\
\hline & & SU 162 & & \\
\hline & & SSU 148 & & \\
\hline & & SSU 147 & & \\
\hline & & NSU $148 \mathrm{~F}$ & & \\
\hline & & NSU $147 \mathrm{~F}$ & & \\
\hline & & SU 154 & & \\
\hline \multirow{2}{*}{8} & \multirow{2}{*}{$\begin{array}{c}\text { PERIODO PARTICO- } \\
\text { SASANIDE? } \\
\text { (presenza di } \\
\text { frammenti di } \\
\text { periodo islamico } \\
\text { fuori contesto?) }\end{array}$} & SU 161 & Monochrome stonepaste ware & $\underline{\text { XII-XIV secolo d.C. }}$ \\
\hline & & SSU 145 & & \\
\hline \multirow{2}{*}{9} & \multirow{2}{*}{ - } & SSU 164 & & \\
\hline & & SSU 155 & & \\
\hline
\end{tabular}

Tab. 1 - Suddivisione in fasi della stratigrafia dell'Area E e principali produzioni ceramiche rinvenute. 


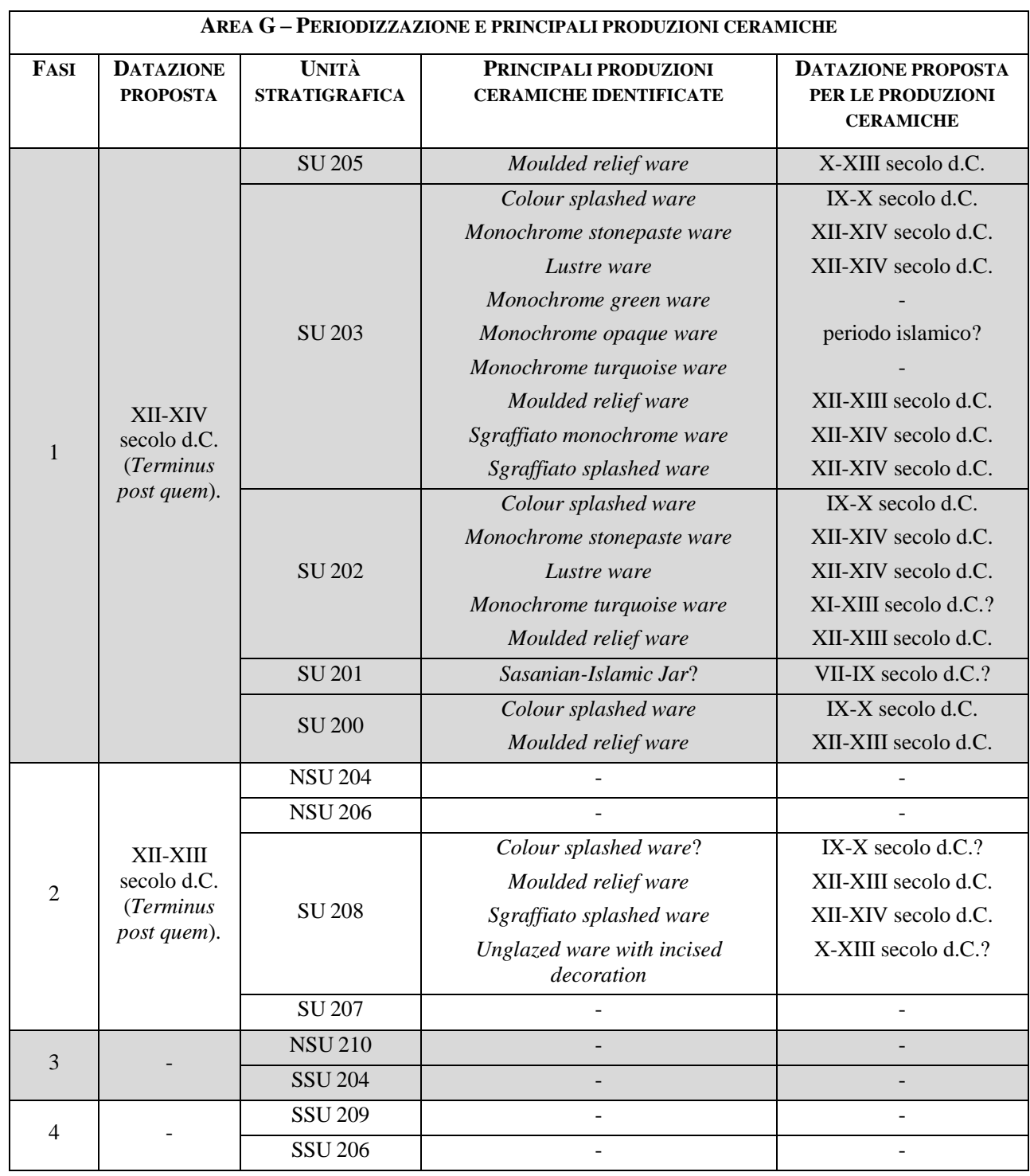

Tab. 2 - Suddivisione in fasi della stratigrafia dell'Area G e principali produzioni ceramiche rinvenute. 


\section{BIBLIOGRAFIA}

Al Yaqoobi, D. - Bogdani, J. - Cereti, C.G. - Colliva, L. - Insom, C. - Terribili, G. - Vecchietti, E. 2015 La Cittadella 2. Fascinazioni dell'antica Erbil, cuore del Kurdistan in Iraq / The Citadel 2. Fascinations of ancient Erbil, heart of Iraqi Kurdistan, Bologna 2015.

Al Yaqoobi, D. - Khorsheed Khader, A. - Mohammed, S. - Hassan Hussein, S. - Shepperson, M. MACGINNIS, J.

2016a Archaeological investigations on the Citadel of Erbil: Background, Framework and Results: K. KopANiAS - J. MACGinNis (eds.) The Archaeology of the Kurdistan Region of Iraq and Adjacent Regions, Oxford 2016, pp. 1-9.

Al Yaqoobi, D. - Michelmore, D. - KhasRaw Tawfiq, R.

2016b Highlights of Erbil Citadel, History \& Architecture. Second Edition (revised), Erbil 2016. Al Yaqoobi, D. - ShePPeRson, M. - MacGinNis, J.

2018 Excavation on the fortifications of the Citadel of Erbil: B. HoREJS - R. SALISBURY - C. SCHWALl - V. MÜLlER - M. LuCIANI - M. RitTER - M. GUIDETTI - F. HÖFLMAYER - T. BÜRGE (eds.), Proceedings of the 10th International Congress on the Archaeology of the Ancient Near East, volume 2, Wiesbaden 2018, pp. 447-460.

BAER, E.

1989 Jeweled Ceramics from Medieval Islam: A Note on the Ambiguity of Islamic Ornament: Muqarnas 6 (1989), pp. 83-97.

BizzArRo, A. - Colliva, L.

2015 Le attivita dell'IsIAO / The activities of IsIAO: D. AL YAQOOBI - A. BIZZARro - M. Bray J. BogdANI - C.G. CeRETI - L. Colliva - M. GAluPPI - C. InSOM - G. LABISI - S. MANCINI G. TERRIBILI (eds.), La Cittadella 2. Fascinazioni dell'antica Erbil, cuore del Kurdistan in Iraq / The Citadel 2. Fascinations of ancient Erbil, heart of Iraqui Kurdistan, Bologna 2015, pp. 109-112.

Bogdani, J. - Colliva, L.

2019 Activities of the Italian Archaeological Mission in Iraqi Kurdistan (MAIKI): P. LuRJE (ed.) Proceedings of the Eighth European Conference of Iranian Studies (State Hermitage Museum and Institute of Oriental Manuscripts, St Petersburg, 14-19 September 2015) (Studies on Pre-Islamic Iran and on Historical Linguistics, vol. I), St Petersburg 2019, pp. 47-65.

Bogdani, J. - Colliva, L. - InSOM, C.

2015 MAIKI. Missione Archeologica Italiana nel Kurdistan Iracheno /MAIKI. Italian Archaeological Mission in Iraqi Kurdistan: D. Al YAQOOBI - A. BIZZARRO - M. BRAY - J. BOGDANI - C.G. CeRETI - L. Colliva - M. GALuPPI - C. InsOM - G. LABISI - S. MANCINI - G. TERribiLi (eds.), La Cittadella 2. Fascinazioni dell'antica Erbil, cuore del Kurdistan in Iraq / The Citadel 2. Fascinations of ancient Erbil, heart of Iraqui Kurdistan, Bologna 2015, pp. 113-126.

2016 Archaeological and Ethnographic Survey in the Paikuli Area (Iraqi Kurdistan): G.L.M. BuRgers - S.J. Kluiving - R.A.E. Hermans (eds.), LAC 2014 Proceedings. Proceedings of the Third International Landscape Archaeology Conference, Rome, 17th - 20th of September 2014, Amsterdam, 2016, pp. 1-11. http://lac2014proceedings.nl/article/view/91.

Cereti, C.G. - Bizzarro, A. - Colliva, L. - Terribili, G.

2013 La Cittadella di Erbil. Le attività del progetto di cooperazione italiano della Sapienza ad Erbil: A. Filipovic - W. Troiano (edd.), Strategie e Programmazione della Conservazione e Trasmissibilità del Patrimonio Culturale, Roma 2013, pp. 222-231. 
Cereti, C.G. - Colliva, L.

2016 Activities of Sapienza-University of Rome in Iraqi Kurdistan: Erbil, Sulaimaniyah and Duhok: K. Kopanias - J. MacGinnis (eds.) The Archaeology of the Kurdistan Region of Iraq and Adjacent Regions, Oxford 2016, pp. 49-56.

Cereti, C.G. - Colliva, L. - Fontana, M.V. - Terribili, G. - Bogdani, J. - Bizzarro, A. - Tilia, A. TILIA, S.S

2012 From Flint to Silicon, Modern Technologies Applied to the Understanding of History. The Italian Archaeological Mission in Iraqi Kudistan: Vicino Oriente XVI (2012), pp. 181-200. Cereti, C.G. - Colliva, L. - Terribili, G.

2019 Il Monumento di Paikuli (Kurdistan - Iraq). Ricerche e Nuove Attività sul Campo: Scienze dell'Antichità 25/1 (2019), pp. 3-10.

CEReti, C.G. - Giunta, R.

2012 Preservation of Cultural Heritage of the Kurdish Region in Iraq, Bologna 2012.

Colliva, L. - TerRibiLi, G.

2017 A forgotten Sasanian sculpture. The fifth bust of Narseh from the monument of Paikuli:

CURTIS, J. Vicino Oriente 21 (2017), pp. 167-195.

2005 The Achaemenid Period in Northern Iraq: P. BRIANT - R. BOUCHARLAT (éd.), L 'archéologie de l'empire achéménide, nouvelles recherches: actes du colloque organisé au Collège de France par le Réseau international d'études et de recherches achéménide (Persika 6), Paris 2005, pp. 175-195.

HANSMAN, J.F.

2011 Arbela (s.v.): Encyclopaedia Iranica Online Edition, 2011, pubblicazione originale: 1986. http://www.iranica.com/articles/arbela-assyrian-arbailu-old.

JENKINS-MADINA, M

2016 Raqqa Revisited: Ceramics of Ayyubid Syria, New York 2016.

Kamal, R.R. - Cereti, C.G. - Colliva, L. - Fusaro, A. - Insom, C. - Labisi, G. - Mancini, S. Terribili, G. - BogDANi, J. - GaLuPPI, M.

2014 MAIKI, Missione Archeologica Italiana nel Kurdistan Iracheno: la carta archeologica dell'area di Paikuli, obiettivi e metodologie applicate: Vicino Oriente XVIII (2014), pp. 2538.

KENNET, D.

2004 Sasanian and Islamic pottery from Ras al-Khaimah: Classification, chronology and analysis of trade in the Western Indian Ocean (British Archaeological Reports International Series 1248), Oxford 2004.

LANE, A.

1957 Later Islamic Pottery: Persia, Syria, Egypt, Turkey (Faber Monographs on Pottery and Porcelain), London 1957 (2nd edition 1971).

LiPPOLIS, C.

2008 Materiali, tecniche costruttive e catalogo degli elementi architettonici dalla Sala Rotonda e dall'Edificio Rosso: A. InvernizZI - C. Lippolis (edd.), Nisa Partica. Ricerche nel Complesso Monumentale Arsacide 1990-2006 (Monografie di Mesopotamia 9), Firenze 2008, pp. 216-268.

MACGINNIS, J.

2014 A City from the Dawn of History: Erbil in the Cuneiform Sources, Oxford 2014. 
MANCINI, S.

2020 Un frammento di moulded relief ware dalla Cittadella di Erbil (Kurdistan iracheno): V. LAVIOLA - M. MASsullo (edd.), Mahabbatnāma, scritti offerti a Maria Vittoria Fontana dai suoi allievi per il suo settantesimo compleanno (Pubblicazioni dell'Istituto per l'Oriente C.A. Nallino 125), Roma 2020, pp. 263-278.

MASON, R.B.

2004 Shine Like the Sun. Lustre-Painted and Associated Pottery from the Medieval Middle East (Bibliotheca Iranica. Islamic art and architecture series 12), Costa Mesa - Toronto 2004.

NoRTHEDGE, A. - KENNET, D

1994 The Samarra Horizon: E.J. Grube (ed.), Cobalt and Lustre. The First Centuries of Islamic NOVÁČEK, K. Pottery (The Nasser D. Khalili Collection of Islamic Art IX), London 1994, pp. 21-35.

2009 Research of the Arbil Citadel, Iraqi Kurdistan, First Season: Subartu 3 (2009), pp. 205-246. PORTER, V.

1981 Medieval Syrian pottery: (Raqqa Ware) (Ashmolean Museum Publications), Oxford 1981. PRIESTMAN, S.M.N.

2021 Ceramic Exchange and the Indian Ocean Economy (AD 400-1275). Volume II: Indian REITLINGER, G. Ocean Pottery Classification (Research Publication 223), London 2021.

1951 Unglazed Relief Pottery from Northern Mesopotamia: Ars Islamica 15-16 (1951), pp. $11-$ 22.

RiIs, P.J. - Poulsen, V. - Hammershaimb, E.

1957 Hama: Fouilles et recherches de la Fondation Carlsberg, 1931-1938. IV.2 Les verreries et RUGIADI, M. poteries médiévales (Nationalmuseets skrifter. Større beretninger 3), Copenhagen 1957.

2011 The Emergence of Siliceous-Paste in Iran in the Last Quarter of the 11th Century and Related Issues. The Dated Assemblage from the Southern Domed Hall of the Great Mosque of Isfahan: Vicino \& Medio Oriente 15 (2011), pp. 233-248.

2016 Stonepaste Technology in Syria and Iran: Sh. CANBy - D. BeyaziT -M. Rugiadi - A.C.S. PeACOCK (eds.), Court and Cosmos. The Great Age of the Seljuqs (New York, The Metropolitan Museum of Art, April 27-July 24, 2016), New York 2016, pp. 179-187, 325SOURDEL, D. 327, 337-354.

1978 Irbil (s.v.): The Encyclopaedia of Islam New Edition, vol. 4, Leiden 1978, pp. 76-77.

UNGER, E.

1929 Arbaïlu (s.v.): E. Ebeling - B. MeIsSNer - E.F. Weidner - W. Von Soden - D.O. EdZARD M.P. Streck - G. FrantZ-Szabò (eds.), Reallexikon der Assyriologie und vorderasiatischen Archäologie 1/3, Berlin 1929, pp. 141-142. 

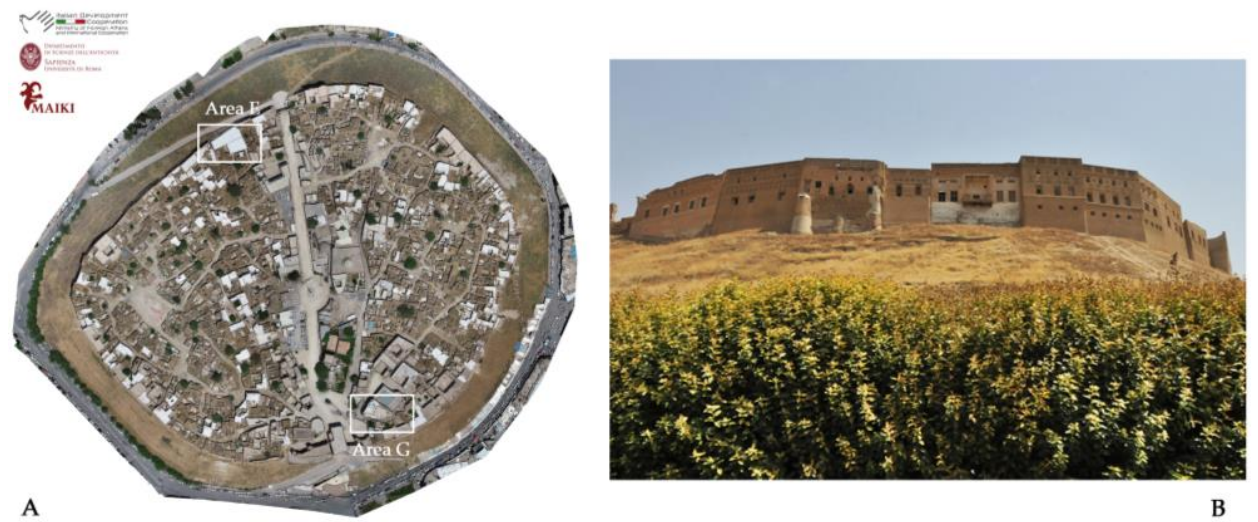

Fig. 1 - La Cittadella di Erbil: a) ortofoto della Cittadella con indicazione delle aree di scavo (ortofoto L. Ebanista-MAIKI, 2019); b) vista da ovest (foto J. Bogdani-MAIKI, 2012).
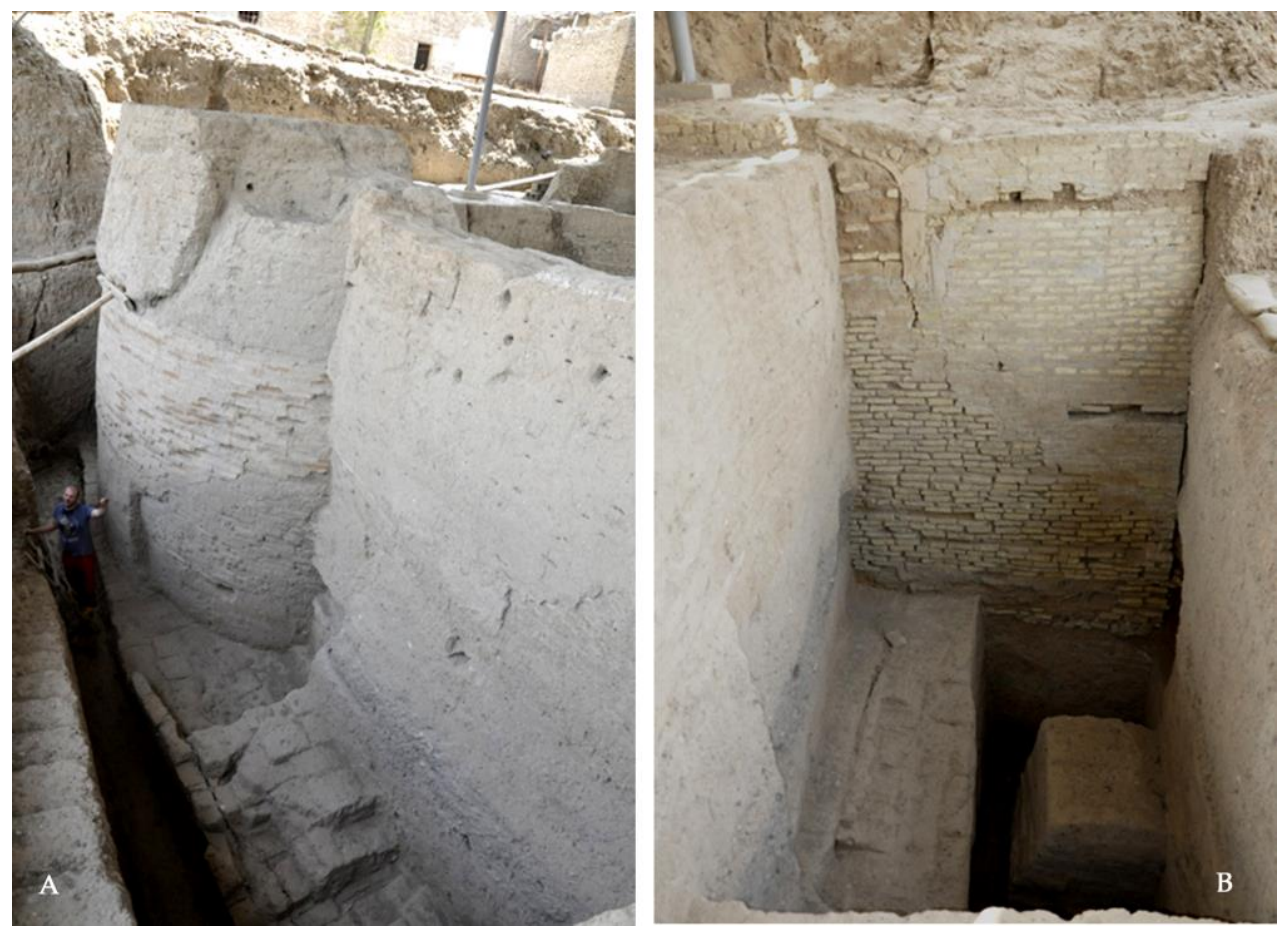

Fig. 2 - Lo scavo dell'Area E: a) la trincea principale; b) l'estensione occidentale (foto L. Colliva-MAIKI, 2016). 


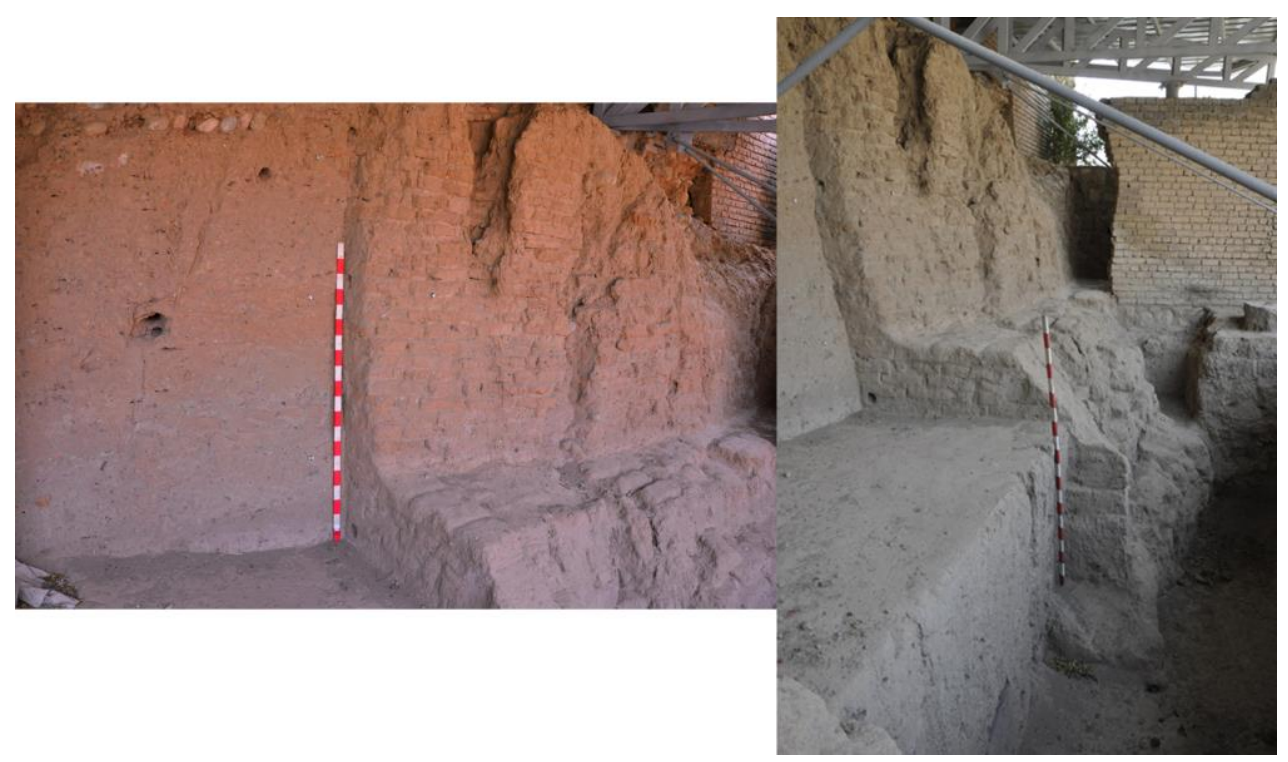

Fig. 3 - Lo scavo dell'Area G (foto M. Galuppi-MAIKI, 2016).
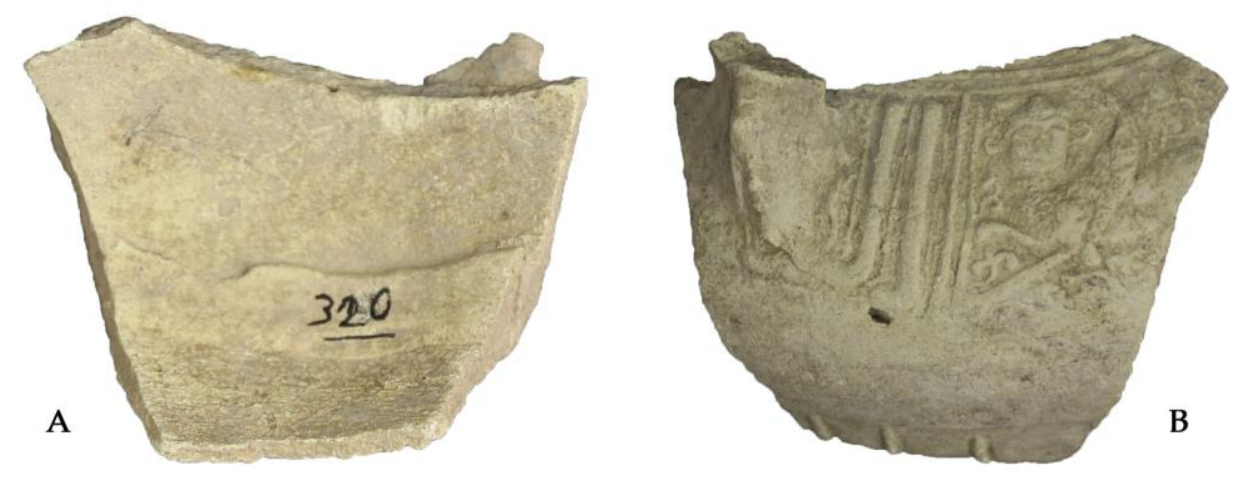

Fig. 4 - Frammento di moulded relief ware: a) superficie interna; b) superficie esterna con decorazione a rilievo realizzata con la tecnica della modellazione a matrice (Foto di S. Mancini-MAIKI, 2017). 


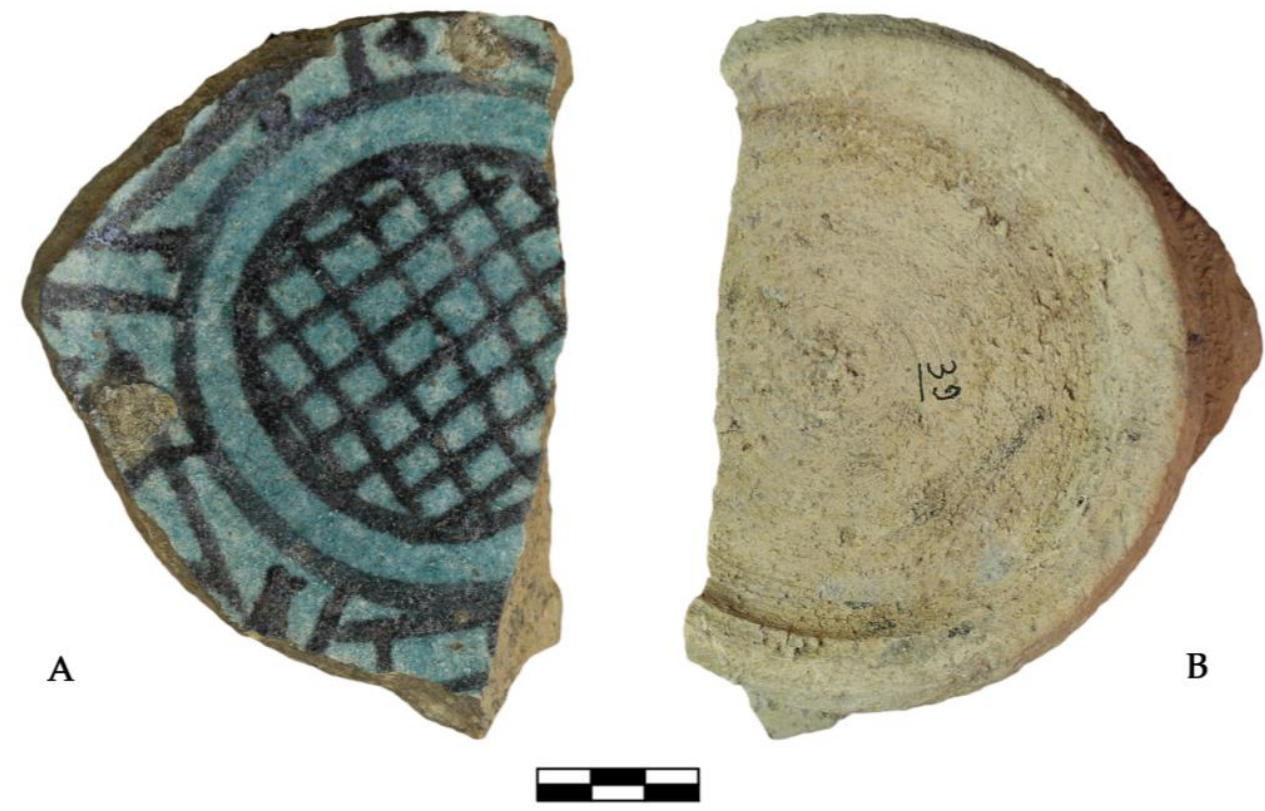

Fig. 5 - Frammento di unglazed black painted ware: a) superficie interna con decorazione dipinta in nero sotto vetrina turchese; b) superficie esterna (Foto di S. Mancini-MAIKI, 2017).

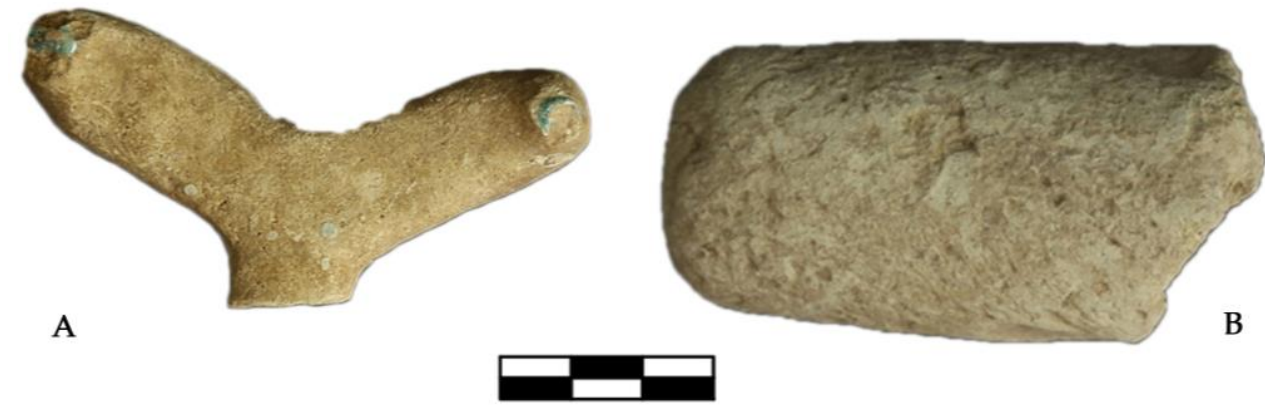

Fig. 6 - a) Frammento di distanziatore con gocce di vetrina (trivet); b) frammento di barra di fornace (kiln peg) (Foto di S. Mancini-MAIKI, 2017). 